\title{
Biomimetics of spider silk spinning process
}

\author{
G. De Luca \& A. D. Rey \\ Department of Chemical Engineering, McGill University, Canada
}

\begin{abstract}
Spiders, with their ultra-optimized spinning process, are able to produce superfibers with remarkable mechanical properties. The precursor material is a lyotropic nematic liquid crystalline anisotropic fluid. The mechanical properties and processability of the silk fiber are intimately connected to the structural transition undergone by this ordered fluid through the spinning pathway. In this work we study a complex mesoscopic structure present in the extrusion duct of spiders' spinning glands, whose stability depends on the interaction between point defects located on the axis of the cavity. The phenomenon described is important in understanding the process-induced structuring of silk fibers and to defect physics in a more general context.
\end{abstract}

Keywords: spider's silk, liquid crystalline spinning, nematic point defects.

\section{Introduction}

Spiders ecologically produce fibers with mechanical properties comparable or superior to the best man-made superfibers $[1,2]$. There is therefore a considerable interest in understanding the design and processing details of the silk-precursor materials. Green spinning processes as well as various exiting applications are envisaged upon the successful mimetic of spider extrusion system and fibers $[3,4,5]$.

Spider silk fibers are spun from a highly concentrated water-based solution of elongated rod-like molecules or aggregates forming a lyotropic nematic liquid crystal phase $[6,7,8]$. This silk precursor can flow as a liquid while maintaining at the same time some degree of orientational order as a crystal. This orientational order is characterized by the tendency that have neighboring rod-like entities to align their long axis in parallel along a common direction $[9,10]$. This preferred molecular orientation usually varies from subregion to subregion in the mesophase (i.e., intermediate phase) due to elastic effects coupled with geometrical and interfacial constraints [9]. The evolution of orientational order or molecular orientation 


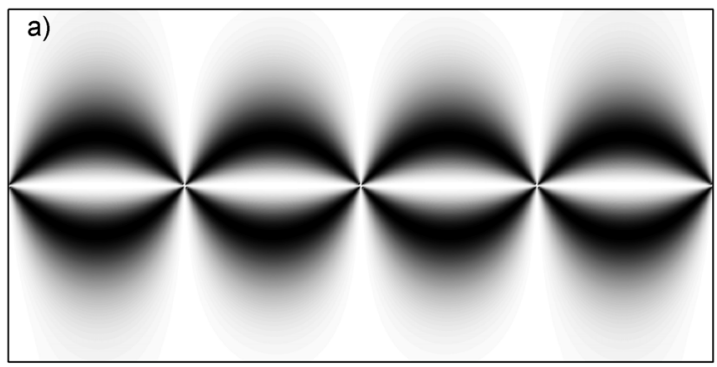

b) I I I I I I I I I I I I I I I I I I I I I I I I I I I I I

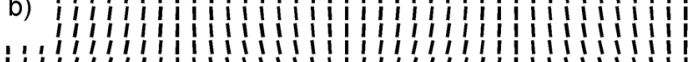

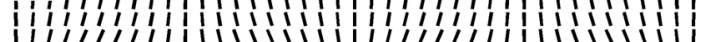

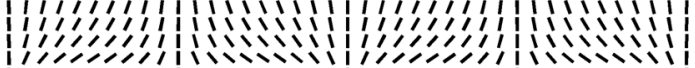

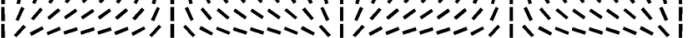

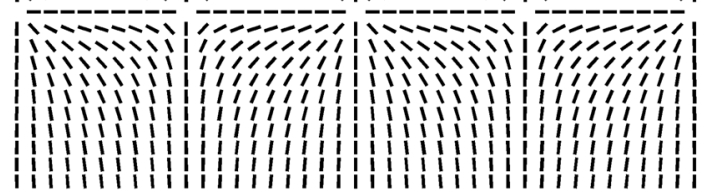

Figure 1: Typical polarized light pattern seen in the extrusion duct of spiders' spinning glands and its correspondence to the ERPD molecular orientation as represented by the director field $\mathbf{n}(\mathbf{r})$.

throughout the spinning pathway is extremely important as it affects the processability of the silk precursor mesophase and determines the microstructural details of the solidified fiber and hence its remarkable mechanical properties $[6,7,8]$.

The spinning apparatus of spiders basically consist of three major regions: a tail where the silk precursor material is synthesized, a central bag where it is stocked in a concentrated solution, and a spinning extrusion duct from which the silk fiber is drawn [11].

Observations made by polarized light microscopy in the extrusion duct have revealed the presence of a complex orientation structure known as escaped radial with point defects (ERPD) [8, 12, 13, 14]. Figure 1 shows a typiolarized light pattern observed along the spinning duct and its corresponding ERPD molecular orientation using the director field representation. The director field $\mathbf{n}(\mathbf{r})$ is given by unit vectors defining local average preferred molecular orientations. Generally $\mathbf{n}(\mathbf{r})=-\mathbf{n}(\mathbf{r})$ and therefore the unit vectors are arrowhead-free $[9,10]$. The point defects, referred as hedgehogs, are located where the direction of bending distortions changes; at those particular locations, no unique director $\mathbf{n}$ can be defined as the orientational order melts. As it can be seen from Fig. 1(b), two types of defects are found in an alternative manner. The point defect found at the center of this figure is of the radial type whereas its two neighbors belong to the hyperbolic type [15]. 
Whether this particular molecular configuration is an accident of Nature or a necessary ingredient of the spider biospinning process is unknown at this time. Nevertheless, it might be hypothesized that this structure may play a role in the control of material crystallization along with water pumping, ions exchanges and $\mathrm{pH}$ reduction phenomena [8]. A premature crystallization of the silk may indeed cause the permanent blockage of the extrusion system and lead to the death of the animal $[8,16]$.

In this work, we investigate the stability of the ERPD structure occurring in the extrusion duct of spiders spinning glands. The stability of the ERPD structure is known to be governed by the interaction between the nematic point defects found along the axis $[15,17]$. This interaction, mediated by the elastic deformations of the material, can cause the defects to move along the axis of the cavity. When two defects of opposite type are sufficiently close to one another they usually annihilate. On the other hand when defects are well separated their interaction is screened [15].

\section{Modeling}

In order to study the stability of the ERPD structure in the extrusion duct of spider we consider two point defects of opposite types located on the axis of cylindrical cavity. Due to the rotational symmetry of the structure, we consider a simple two dimensional rectangular domain representing half of a cross section. Dimensionless quantities (denoted by overbars) and equations are used to reduced the number of parameters and facilitates analysis as well as comparisons. The dimensionless width and height of the computational domain correspond respectively to the dimensionless length $\bar{Z}$ and radius $\bar{R}$ of the cylindrical cavity. The dimensionless position vector is defined as $\overline{\mathbf{r}}=\mathbf{r} / R$ where $R$ is the dimensional radius of the cavity .

The continuum nemato-dynamics equation describing the structure evolution of a nematic liquid crystal is typically derived from the minimization of a free energy functional depending on some order parameter [9]. In this work the nematic ordering is described in terms of a tensor field $\mathbf{Q}(\mathbf{r})$, called tensor order parameter [9]. This tensor order parameter is symmetric traceless (i.e., $Q_{i j}=Q_{j i}$ and $Q_{i i}=$ 0 ) and, according to a spectral decomposition, reads:

$$
Q_{i j}=\mu_{n} n_{i} n_{j}+\mu_{m} m_{i} m_{j}+\mu_{l} l_{i} l_{j}
$$

In this expression, $\mathbf{n}, \mathbf{m}$ and $\mathbf{l}$ are unit eigenvectors forming an orthogonal triad and $\mu_{n}, \mu_{m}$ and $\mu_{l}$ are their corresponding eigenvalues. The director triad and the eigenvalues are characterizing the orientation and the strength of alignment of the phase respectively. The largest eigenvalue in magnitude or absolute value, $\mu_{n}$, gives the strength of ordering along the uniaxial director $\mathbf{n}$ previously defined. The second $\mu_{m}$ and third $\mu_{l}$ eigenvalues correspond respectively to the biaxial directors $\mathbf{m}$ and $\mathbf{l}(\mathbf{l}=\mathbf{n} \times \mathbf{m})$. At equilibrium, an undistorted nematic phase is uniaxial; however, in distorted regions like in the vicinity of defects the phase may exhibit some biaxiality [9]. 
The eigenvalues $\mu_{i}(i=1,2,3)$ of the tensor order parameter are restricted by: $-1 / 3 \leq \mu_{i} \leq 2 / 3$ and $\mu_{n}+\mu_{m}+\mu_{l}=0$. The ordering states described by the tensor order parameter are: isotropic $\left(\mu_{n}=\mu_{m}=\mu_{l}=0 ; \mathbf{Q}=0\right)$, uniaxial $\left(\mu_{n}>\mu_{m}=\mu_{l}\right)$ and biaxial $\left(\mu_{n} \neq \mu_{m} \neq \mu_{l}\right)$. Finally it is stressed that within this tensorial formalism, order is continuous and defined everywhere including at defect cores unlike in the simpler vectorial description in terms of the director field (See Fig. 1).

It is also often useful to represent the tensor order parameter $\mathbf{Q}$ in the following alternative, but more compact format:

$$
Q_{i j}=S\left(n_{i} n_{j}-\frac{\delta_{i j}}{3}\right)+\frac{P}{3}\left(m_{i} m_{j}-l_{i} l_{j}\right)
$$

In this expression, $S$ and $P$ are uniaxial and biaxial scalar order parameters describing respectively the strength of alignment around the uniaxial and biaxial directors (i.e., $\mathbf{n}$ and $\mathbf{m}$ ). The Kronecker $\boldsymbol{\delta}$ stands for the unit tensor. The scalar order parameters are defined as: $S=3 / 2\left(n_{i} Q_{i j} n_{j}\right)$ and $P=3 / 2\left(m_{i} Q_{i j} m_{j}-l_{i} Q_{i j} l n_{j}\right)$. The correspondence between the scalar order parameters and the eigenvalues is as follow: $\mu_{n}=2 / 3 S, \mu_{m}=-1 / 3(S-P)$ and $\mu_{m}=-1 / 3(S+P)$. The scalar order parameters obey the following restrictions: $-1 / 2 \leq S \leq 1$ and $-3 / 2 \leq P \leq 3 / 2$.

The dimensionless total free energy of a nematic liquid crystal $\bar{F}$ system under strong anchoring conditions (i.e., the orientation of the molecules at the boundary is fixed) is generally written as [9]:

$$
\bar{F}=\int_{V}\left(\bar{f}_{h}+\bar{f}_{g}\right) d \bar{r}^{3}
$$

In this expression, $\bar{f}_{h}$ and $\bar{f}_{g}$ represent respectively the homogeneous and gradient bulk free energies. We note that $\bar{f}_{b}=f_{b} / A$ where $\bar{f}_{b}=\bar{f}_{h}+\bar{f}_{g}$ and $A$ is an energy density scale. Also, accordingly $\bar{F}=\frac{F}{A R^{3}}$. The homogeneous free energy describes the short-range ordering effects related to the amplitude of the tensor order parameter. This expression permits to describe the first order isotropicnematic phase transition but also the variation of ordering in the vicinity of defects. This contribution is often referred as the Landau-de Gennes free energy. According to Doi's formalism [18], this expension of the order parameter may be written, in dimensionless form, as:

$$
\bar{f}_{h}=\frac{1}{2}\left(1-\frac{U}{3}\right) Q_{i j} Q_{j i}-\frac{U}{3} Q_{i j} Q_{j k} Q_{k i}+\frac{U}{4}\left(Q_{i j} Q_{j i}\right)^{2}
$$

In this expression $U$ is a dimensionless phenomenological parameter called nematic potential which controls the magnitude of the equilibrium tensor order parameter. In general the nematic potential $U$ can be assigned a dependence on either temperature or concentration depending on the nature of the nematic liquid crystal (i.e., thermotropic or lyotropic) [18]. Within this format, the first order phase isotropic-nematic phase transition occurs at nematic potential $U_{I N}=2.7$. Also, the system is isotropic for $U<U_{I N}$ and nematic for $U>U_{I N}$. The limit 
of metastability for the isotropic and nematic phase are $U^{*}=3$ and $U^{* *}=8 / 3$, respectively.

The gradient bulk free energy $\bar{f}_{g}$ represents the energy variations due to longrange ordering effects. This energy penalty associated with the elastic distortions of the phase can be expressed in dimensionless form as:

$$
\bar{f}_{g}=\frac{\bar{\xi}^{2}}{2} \bar{\nabla}_{k} Q_{i j} \bar{\nabla}_{k} Q_{i j}
$$

In this equation $\bar{\xi}$ represents the reduced nematic coherence length which gives a characteristic scale for the variation of the tensor order parameter and the size of defect cores or thickness of the nematic-isotropic interface. It is noticed that $\bar{\nabla}=R \nabla, \bar{\xi}=\xi / R$ and $\xi=\sqrt{L / A}$ where $L$ a material-specific elastic constant.

The dynamic equation describing the relaxation of the tensor order parameter $\mathbf{Q}(\mathbf{r}, t)$ towards an equilibrium value that minimizes the total free energy $\bar{F}$ follows from variational principles and is given in dimensionless format by [19]:

$$
\frac{\partial Q_{\alpha \beta}}{\partial \bar{t}}=-\frac{\delta \bar{F}}{\delta Q_{\alpha \beta}}
$$

The right-hand side of this expression corresponds to functional derivative of the of the total free energy. Here again we stress that $\bar{t}=\frac{A}{\gamma} t$ where $\gamma$ a kinetic constant associated with rotational viscosity. From variational calculus it can be shown that:

$$
\frac{\delta \bar{F}}{\delta Q_{\alpha \beta}}=\frac{\partial \bar{f}_{b}}{\partial Q_{\alpha \beta}}-\bar{\nabla}_{\gamma} \frac{\partial \bar{f}_{b}}{\partial \bar{\nabla}_{\gamma} Q_{\alpha \beta}}
$$

Only the symmetric traceless part of this expression is retained in order to satisfy the constraints of the tensor order parameter.

The boundary conditions for the problem are as follows: at the wall of the cylindrical cavity (upper part of the rectangular domain), the tensor order parameter is assumed to be uniaxial and describe a strong radial anchoring condition so that $Q_{i j}(r=R)=S_{e}\left(e_{i}^{r} e_{j}^{r}-\frac{\delta_{i j}}{3}\right)$, where $e^{r}$ is the unit vector along the radial direction. The equilibrium scalar order parameter $S_{e}$ is obtained analytically from Eq. 4 and is given by the relation [18]:

$$
S_{e}=\frac{1}{4}+\frac{3}{4} \sqrt{1-\frac{8}{3 U}}
$$

On the $z$-axis (lower part of the rectangular domain), rotational symmetry boundary condition is assumed. Finally, on the sides of the domain, Neumann conditions are enforced so as to emulate an infinitely long cavity.

Initially, the systems contains a hedgehog pair whose cores are separated by a distance $\bar{D}=2.4$. The hyperbolic and radial hedgehogs are respectively located at $\bar{z}=-1.2$ and $\bar{z}=1.2$. The corresponding initial tensor field $\mathbf{Q}(\mathbf{r}, t=0)$ is obtained by taking a few time steps starting from a trial configuration satisfying 
(a)

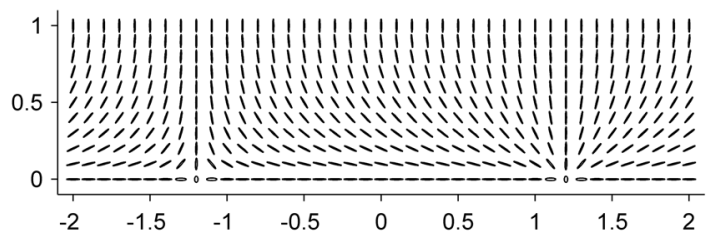

(b)

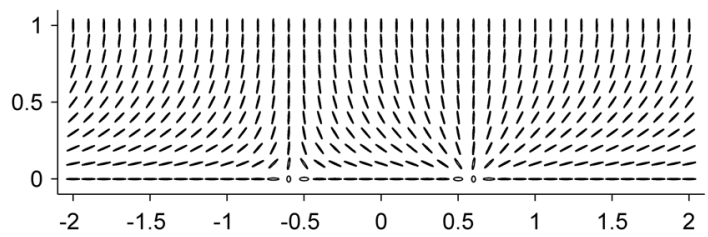

(c)

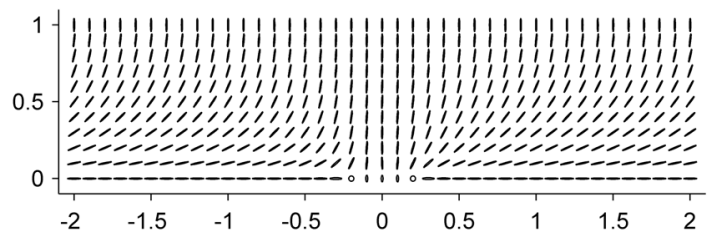

(d)

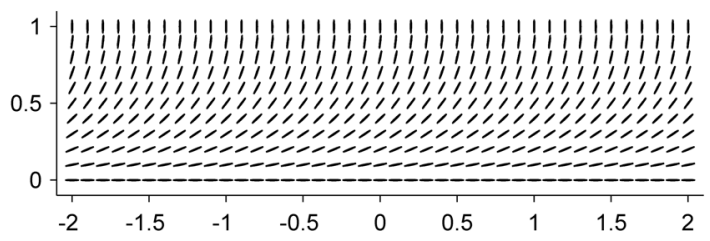

Figure 2: Evolution of orientational order during the annihilation of two nematic point defects confined in a cylindrical capillary. Each ellipse represents a tensor order parameter $\mathbf{Q}$. The orientation and amplitude of an ellipse is given by the eigenvectors and eigenvalues of $\mathbf{Q}$. (a) $\bar{t}=0$, (b) $\bar{t}=39740$, (c) $\bar{t}=40600$ and (d) $\bar{t}=40900$.

all the boundary conditions. Other initial defect configurations do not change the essential features of the results.

The model used in this work contains two parameters: the nematic potential $U$ and the reduced nematic coherence length $\bar{\xi}$. For all the simulation results presented in this paper, the nematic potential is set to $U=6$ which corresponds to an equilibrium scalar order parameter of $S_{e}=0.809$. Other values of $\mathrm{U}$ in the stable nematic range do not change the underlying process under study. The value of the reduced coherence length is fixed at $\bar{\xi}=1 / 30$. 


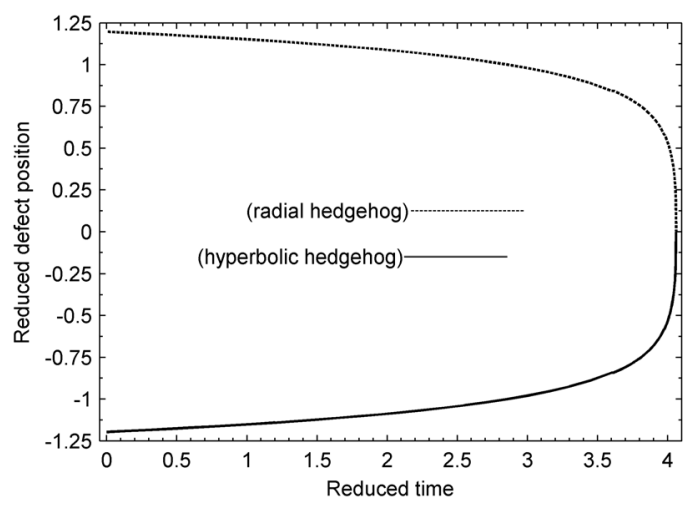

Figure 3: Position of the point defects along the cavity axis as a function of time.

The governing dynamic equation for the tensor order parameter $\mathbf{Q}(\overline{\mathbf{r}}, \bar{t})$ is solved using the standard numerical method of lines. The space discretization is achieved by the finite element method. The time integration of the resulting differential equations is obtained using an adaptive implicit scheme. The density of element is higher in the region describing the trajectories of the defects along the $\bar{z}$-axis. The independence of solutions on mesh density was verified using standard procedures. The size of the triangular elements is always smaller than the reduced coherence length $\bar{\xi}$ (smallest length in the problem) to accurately capture the amplitude variations of the tensor order parameter. The reduced width and height of the computational domain are respectively $\bar{Z}=6$ and $\bar{R}=1$.

\section{Results}

This section illustrates the dynamic interaction between two nematic hedgehogs confined in a capillary tube. A similar process is though to occur in the extrusion duct of spider's spinning gland. Figure 2 shows the typical evolution of orientational order during the annihilation of the point defect pair. In this figure, the tensor order parameter $\mathbf{Q}(\mathbf{r}, t)$ is represented as an ellipse. The orientation and amplitude of each ellipse is given by the eigenvectors and eigenvalues of the order parameter. Within, this representation, an elongated ellipse corresponds to a well-ordered nematic region whereas a circle corresponds to a low orientational ordering. Three stages of the annihilation process are shown. In the pre-collision regime Fig. 2(a) and Fig. 2(b), the two point defects have distinct, well separated, cores. As they move closer one another, the distortion in the ellipses orientation increased. However, this process is not accompanied by any significant changes in the defect core structure and ordering. In the collision regime Fig. 2(c), the cores of the defects slowly overlap to finally become indistinguishable. In this regime, structural changes are mainly given by changes in alignment (i.e., amplitude of the 


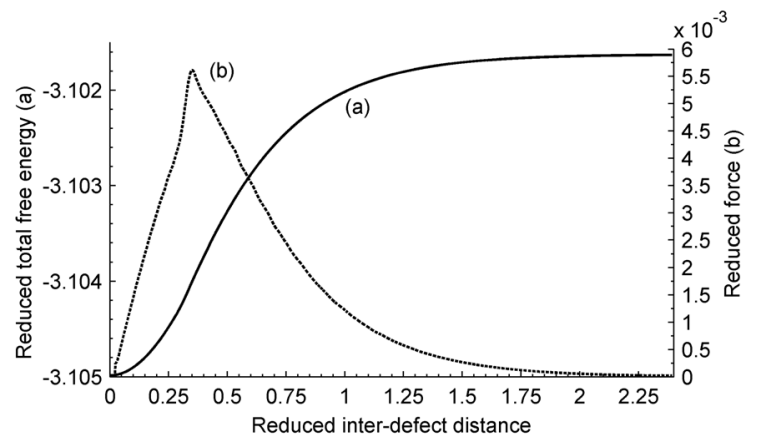

Figure 4: Total free energy and interaction force between the point defects as function of the inter-defect distance.

tensor) rather than orientation. It can be seen that the shape of the ellipses now changes in the inter-defects region in contrast to the pre-collision regime. Finally in the post-collision regime Fig. 2(d), the tensor order parameter field relaxes to its equilibrium value and the remaining axial gradients are smoothed-out. The resulting structure, known as escape radial (ER), is defect free and possesses a single bending direction.

Figure 3 illustrates the displacement of the two point defects during the annihilation process. The positions of the defects correspond to the minima of the uniaxial scalar order parameter $S$. The two trajectories show that the defects travel at identical speeds. The speed of each defect is however not constant and tend to dramatically increase as the defects approach each other. At large separating distance, the defects speed is essentially null.

Figure 4 shows the variation of the total free energy as a function of the defect separating distance and corresponding interaction force. The interaction force is computed by differentiating the total free energy with respect to the inter-defect distance. It can be seen from this figure that the interaction force tends to zero as defects are separated by more than a diameter. However as defects approach each other, this interaction force increases exponentially. In the late stage of the collision regime, governed by alignment and therefore the amplitude of the tensor order parameter, the interaction force is decreasing in a quasi-linear fashion. The value of the force at vanishing defect separating distance is small but finite to the level of discretization of our model.

\section{Conclusions}

Motivated by the reported experimental observation of ERPD structures in the extrusion duct of spiders spinning apparatus, we have investigated numerically the dynamic interaction between two nematic point defects of opposite topological charge confined in cylindrical cavity. In contrast to previous analytical [17] 
and numerical [15] studies we employed the tensor order parameter formalism to describe the orientational order of the nematic phase, which allows for an unambiguous description of defects and reliable estimation of energies. Three distinct regimes leading to the annihilation of the antagonist point defect pair were described. The trend of the defect trajectories agree very well the one found in theoretical [17] and experimental [20] studies. The absence of asymmetry in the defect trajectories as reported in Ref. [21] is attributed to the absence of backflow effects as well as to the isotropy of the elastic constant. These effects will be investigated in future work and will be reported. The reasons of the point defect annihilation were also investigated. In particular, the dependence of the total free energy and corresponding interaction force on the inter-defect distance was analyzed. It was shown that the interaction force between the defect, which set them into motion, is decreasing exponentially at large defect separating distance. As predicted theoretically in Ref. [22] and shown experimentally if the point defects are separated by a distance greater than a diameter, the interaction force is shielded and the defects pinned. In contrast to previous studies having reported explicitly the force of interaction, we have found that at short separating distance, the interaction force was decreasing very steeply in a linear way. We show that this distinctive behavior is due to the significant variation of the order parameter in the late stage of the collision regime. During the whole process the interaction force was found to be strictly attractive. Finally we would like to emphasize that despite that the context of our study is the process-induced structure in the biospinning of spider silk, the results obtained should be useful to the field of defect physics. The present work provides a theoretical framework to eventually extract engineering principles used in the biospinning process. Having established the stability properties of the structural unit (pair of point defects) in the spider duct, the next step will be to elucidate the emergence of this structural unit and its interaction with other neighboring point defects, as they arise in real spider ducts. This work is supported by the Natural Science and Engineering Research Council of Canada (NSERC). G.D. wishes to acknowledge financial support from NSERC through the CGS program.

\section{References}

[1] Gosline, J., DeMont, M. \& Denny, M., The structure and properties of spider silk. Endeavour, 10(1), pp. 37-43, 1986.

[2] Ko, F. \& Jovicic, J., Modeling of mechanical properties and structural design of spider web. Biomacromolecules, 5, pp. 780-785, 2004.

[3] Atkins, E., Silk's secrets. Nature, 424, p. 1010, 2003.

[4] Jin, H.J. \& Kaplan, D., Mechanism of silk processing in insects and spiders. Nature, 424, pp. 1057-1061, 2003.

[5] Turner, J. \& Karatzas, C., Natural Fibers, Plastics and Composites, Kluwer Academic Publishers, chapter 1, Advanced spider silk fibers by biomimicry, pp. 11-23, 2004.

[6] Kerkam, K., Viney, C., Kaplan, D. \& Lombardi, S., Liquid crystallinity of natural silk secretions. Nature, 349, pp. 596-598, 1991. 
136 Design and Nature III: Comparing Design in Nature with Science and Engineering

[7] Viney, C., Huber, A., Dunaway, D., Kerkam, K. \& Case, S., Silk Polymers: Materials Science and Biotechnology, American Chemical Society, chapter 11, Optical Characterization of Silk Secretions and Fibers, pp. 120-136, 1993.

[8] Vollrath, F. \& Knight, D., Liquid crystalline spinning of spider silk. Nature, 410, pp. 541-548, 2001.

[9] de Gennes, P. \& Prost, J., The Physics of Liquid Crystals. Oxford University Press, 1995.

[10] Collings, P., Liquid Crystals: Nature's Delicate Phase of Matter. Princeton University Press, 2001.

[11] Foelix, R., Biology of spiders. Oxford University Press, 1996.

[12] Knight, D. \& Vollrath, F., Liquid crystals and flow elongation in a spider's silk production line. Proc R Soc Lond B, 266, pp. 519-523, 1999.

[13] Vollrath, F., Strength and structure of spiders' silks. Reviews in Molecular Biotechnology, 74, pp. 67-83, 2000.

[14] Lydon, J., Silk: the original liquid crystalline polymer. Liquid Crystals Today, 13(3), pp. 1-13, 2004.

[15] Vilfan, I., Vilfan, M. \& Zumer, S., Defects structures of nematic liquid crystals in cylindrical cavities. Physical Review A, 43(12), pp. 6875-6880, 1991.

[16] Wainwright, S., Biggs, W., Currey, J. \& Gosline, J., Mechanical Design in Organisms. Princeton University Press, 1982.

[17] Peroli, G. \& Virga, E., Annihilation of point defects in nematic liquid crystals. Physical Review E, 54(5), pp. 5235-5241, 1996.

[18] Doi, M., The Theory of Polymer Dynamics, 1998.

[19] Beris, A. \& Edwards, B., Thermodynamics of Flowing Systems. Oxford University Press, 1994.

[20] Peroli, G., Hillig, G., Saupe, A. \& Virga, E., Orientational capillary pressure on a nematic point defect. Physical Review E, 58(3), pp. 3259-3263, 1998.

[21] Cladis, P. \& Brand, H., Hedgehog-antihedgehog pair annihilation to a static soliton. Physica A-Statistical Mechanics And Its Applications, 326(3-4), pp. 322-332, 2003.

[22] Semenov, A.N., Interaction of point defects in a nematic liquid. Europhysics Letters, 46(5), pp. 631-636, 1999. 